\title{
Nanoscale molecular communication networks: a game-theoretic perspective
}

\author{
Chunxiao Jiang $^{1 *}$, Yan Chen² and KJ Ray Liu
}

\begin{abstract}
Currently, communication between nanomachines is an important topic for the development of novel devices. To implement a nanocommunication system, diffusion-based molecular communication is considered as a promising bio-inspired approach. Various technical issues about molecular communications, including channel capacity, noise and interference, and modulation and coding, have been studied in the literature, while the resource allocation problem among multiple nanomachines has not been well investigated, which is a very important issue since all the nanomachines share the same propagation medium. Considering the limited computation capability of nanomachines and the expensive information exchange cost among them, in this paper, we propose a game-theoretic framework for distributed resource allocation in nanoscale molecular communication systems. We first analyze the inter-symbol and inter-user interference, as well as bit error rate performance, in the molecular communication system. Based on the interference analysis, we formulate the resource allocation problem as a non-cooperative molecule emission control game, where the Nash equilibrium is found and proved to be unique. In order to improve the system efficiency while guaranteeing fairness, we further model the resource allocation problem using a cooperative game based on the Nash bargaining solution, which is proved to be proportionally fair. Simulation results show that the Nash bargaining solution can effectively ensure fairness among multiple nanomachines while achieving comparable social welfare performance with the centralized scheme.
\end{abstract}

Keywords: Nanocommunication; Molecular communication; Game theory; Non-cooperative game; Cooperative game; Nash bargaining

\section{Introduction}

Nanotechnology, a manipulation of matter on an atomic and molecular scale, makes the design and fabrication of nanoscale components become a reality. Such nanoscale components can be used to assemble basic structural and functional devices, called nanomachines, which are able to perform basic and simple tasks at the nanolevel, such as computing, data storing, sensing, and actuation [1]. Recently, this promising technology catalyzes a new communication paradigm - 'nanocommunications,' arousing the researchers' great interests in both industrial and academic fields. Nanocommunications refer to the information exchange between nanomachines, which can be realized through nanomechanical, acoustic, electromagnetic, and chemical or molecular communication means.

*Correspondence: jchx@tsinghua.edu.cn

${ }^{1}$ Department of Electronic Engineering, Tsinghua University, Beijing 100084, China

Full list of author information is available at the end of the article
It is expected that nanocommunication networks can be applied in many different scenarios including human health monitoring, food and water quality control, air pollution control, as well as aggressive chemical agent detection [2].

Since molecule migration naturally occurs within both living organisms and abiotic components, molecular communication is considered as the most promising approach for nanocommunication networks [3,4], where the transmission and reception of information are realized through molecules. In the molecular communication model, the nanotransmitter releases molecules, which are modulated and coded to carry information, into the fluid medium. The molecules propagate to the receiver through the medium, which are demodulated and decoded to restore the information that the transmitter intends to convey. There are mainly three molecule prorogation models: walkway-based model, flow-based model, and diffusion-based model [5]. In the walkway-based model,

\section{望 Springer}

C 2015 Jiang et al.: licensee Springer. This is an Open Access article distributed under the terms of the Creative Common Attribution License (http://creativecommons.org/licenses/by/4.0), which permits unrestricted use, distribution, and reproduction in any medium, provided the original work is properly credited. 
the molecules propagate through physical pathways connecting the transmitter to the receiver, such as molecular motors [6]. In the flow-based model, the propagation of the released molecules is controlled by the predefined flow and turbulence in the medium, such as pheromonal communication [2]. In the diffusion-based model, the molecules propagate through their spontaneous diffusion in the fluid medium, such as calcium signaling among cells [7]. In this paper, we focus on the diffusion-based model since it represents the most general and widespread molecular communication architecture found in nature.

In the literature, various topics about the diffusionbased molecular communications have been studied, including channel model [8-13], modulation and coding [14-18], and receiver design [19,20]. The earlier works regarding diffusion-based molecular communications were mainly focused on channel capacity analysis [8-13], where a commonly accepted channel model is based on Brownian motion [21]. In [8], Eckford analyzed the achievable bound on information rate for the diffusion-based channel with one-dimensional Brownian motion. As an extension, Brownian motion of molecules in a fluid medium with drift velocity instead of static environment was analyzed in [13]. In addition to the channel analysis, a comprehensive physical end-to-end model, including molecule emission, diffusion, and reception, was proposed in [5]. Meanwhile, simulation-based approaches for exploring the diffusive molecules were conducted in [22] and [23]. As for channel noise and interference analysis, Pierobon and Akyildiz studied molecule sampling and counting noise in [16], as well as the inter-symbol and co-channel interference in [17]; Kadloor et al. presented an additive inverse Gaussian noise channel model in [14]. To enhance system performance, various coding schemes were also introduced, including a forward error correction coding scheme in [15] and a rate-delay trade-off network coding scheme in [18]. In terms of receiver design, an optimal receiver design based on weighted sum detectors was proposed in [19], and a ligand-binding reception model was studied in [20]. Moreover, the consensus problem and relaying role under diffusion-based molecular communication were recently studied in [24] and [25], respectively.

However, the resource allocation issue in molecular communication networks has not yet been investigated. In traditional electromagnetic wave wireless communications, when multiple users share the same resource, e.g., power and spectrum, how to allocate the resource among different users is very important to guarantee the high system efficiency while maintaining fairness $[26,27]$. Similarly, in the diffusion-based molecular communications, there can be multiple transmitters sharing the same fluid medium. In such a case, interference will occur since the molecules from different transmitters are identical and indistinguishable [13]. Therefore, how to perform resource allocation among multiple transmitters, molecule emission control, is an essential problem in practical molecular communication systems. Generally, the computational capability of nanomachines is low, and the information exchange cost among them is expensive. Thus, distributed resource allocation algorithms are strongly favorable in molecular communications. Moreover, different nanomachines may be fabricated by different manufacturers and may have different objectives, e.g., in human body monitoring nanosensor networks [3], different biomedical sensors may have different functionalities and may be deployed by different doctors. Considering these problems, in this paper, we propose a game-theoretic framework for distributed resource allocation in diffusion-based molecular communications. The game-theoretical model provides distributed molecule emission control solutions to achieve high system efficiency, while guaranteing fairness among different nanomachines. Game theory has been corroborated as an effective tool for modeling different problems in traditional distributed wireless communication networks [28]. To the best of our knowledge, this is the first work that uses game theory to study the interactions among nanomachines in molecular communication networks, which is expected to exemplify the application of game theory in the nanocommunication and networking field.

The contributions of this paper can be summarized as follows.

1. We analyze the inter-symbol and inter-user interference in the molecular communication system with amplitude modulation. Based on the interference analysis, we find the optimal decision threshold using the maximum-a-posterior (MAP) detection method and derive the corresponding bit error rate (BER) performances of different transmitters.

2. We propose a game-theoretic framework to model the resource allocation problem in nanoscale molecular communication systems. Specifically, we focus on the molecule emission control issue by using a two-transmitter and one-receiver system as an example. In the proposed game-theoretic framework, the players are the transmitters whose objectives are to maximize their own utilities, the strategy of each player is the number of emitted molecules, and the utility function is related with the BER performance.

3. Based on the game-theoretic framework, we formulate the molecule emission control problem as a non-cooperative emission control game, where the Nash equilibrium (NE) is derived and proved theoretically as the unique NE. In order to improve the system efficiency while guaranteeing fairness, we 
further model the problem using a cooperative game based on the Nash bargaining solution, where we prove that the Nash bargaining solution of the proposed emission control game is a proportionally fair solution.

The rest of this paper is organized as follows. We first introduce the system model of molecular communication in Section 2. Then, we analyze the inter-symbol and inter-user interference and BER performance in Section 3. The non-cooperative and cooperative molecule emission control games with simulation results are discussed in Section 4. Finally, conclusions are drawn in Section 5.

\section{System model}

\subsection{Network entity}

As shown in Figure 1, in this paper, we consider a molecular communication system with two transmitters and one receiver. However, we would like to point out that since the interference from different transmitters is additive due to the independence among transmitters, all the analyses and results in this paper can be easily extended to the multi-transmitter and multi-receiver case. Moreover, the malicious nanonodes are not taken into account in this paper. As we can see from Figure 1, there are four components in a diffusion-based molecular communication system: transmitter, molecule, propagation medium, and receiver, whose functionalities are described as follows.

- Transmitter: Each transmitter can independently generate and emit molecules into the propagation medium. It is responsible for the modulation of a series of input symbols $s(t) \in\{0,1\}$ by controlling the speed, number, or time of the emitted molecules. We assume that the transmitters can perfectly control the release time and number of the molecules, while having no control on the motion of the molecules once the molecules escape from the transmitter [5,9].

- Molecule: A molecule is an indivisible object that can be released by the transmitter and absorbed by the receiver by means of chemical reactions. The molecules carry information of the transmitters and randomly diffuse in the propagation medium. They are considered as identical and undistinguishable between each other. Moreover, the interactions among molecules are not taken into account in general [14]. Therefore, the trajectories of all molecules in the medium are independent of each other.

- Propagation medium: The propagation medium is made of some kind of fluid, where the molecules can freely diffuse inside. Relative to a single molecule, the space of the medium is considered as infinite in any dimension. The Brownian motion is a generally accepted model for the propagation of molecules in the medium, which can be characterized by two parameters: drift velocity and diffusion constant determined by the physical properties of the fluid medium [29]. In this paper, we only consider the diffusion effect of molecules. Note that [13] considered both diffusion and drift effects, and all our analyses and results can be easily extended to that case.

- Receiver: When the molecules arrive at the receiver, they are absorbed by the receiver and disappear from the medium. It is assumed that the receiver can perfectly measure the time when it absorbs a single molecule and use such information to determine the

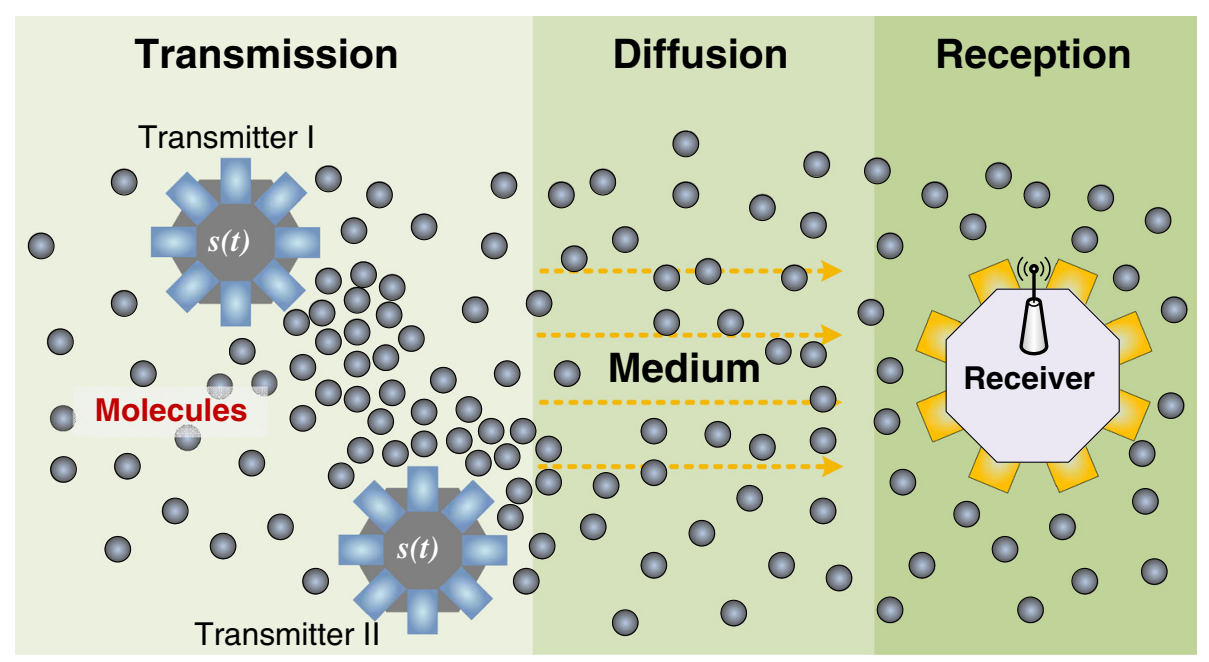

Figure 1 System model with two transmitters and one receiver. 
information sent by the transmitter. Moreover, the synchronization between the transmitter and receiver is also assumed to be perfect [30-32]. Note that the focus of this paper is the interference and resource allocation in molecular communication networks, while the synchronization issue is out of the scope. In molecular communication networks, the receiver is usually more powerful than the transmitter, having a larger size, which is usually designed to be able to communicate with microscale machines using electromagnetic waves as well.

\subsection{Modulation}

At the transmitter, the input symbols $s(t)$ can be modulated onto the molecules with different approaches:

- Frequency modulation: controlling the density/speed of the emitted molecules [5]

- Timing modulation: controlling the emission time of the molecules [14]

- Amplitude modulation: controlling the number of the emitted molecules [15]

In this paper, we consider the amplitude modulation. Specifically, at the beginning of each time slot, the transmitter releases $n$ molecules to represent transmitting bit ' 1 ' and releases 0 molecule to represent transmitting bit ' 0 ', i.e., only one bit transmission per time slot, as shown in Figure 2. The length of each time slot $t_{s}$ is considered as identical for all transmitters. For the reception side, the receiver counts and accumulates the number of absorbed molecules during each time slot, comparing the number with some predefined threshold to make a decision on receiving bit ' 1 ' or ' 0 '. In such a case, the number of molecules emitted at time slot $t, e(t)$, is

$$
e(t)=s(t) \cdot n,
$$

where $s(t)$ is a series of ' $0-1$ ' symbols. Note that $n$ can be regarded as the molecule emission power.

\subsection{Channel model}

In molecular communication systems, the communication channel is the fluid medium and the propagation model is based on the Brownian motion [21]. Due to the random behavior of the molecules with Brownian motion, after escaping from the transmitter, the time they arrive at the receiver is probabilistic. Suppose the transmitter is located at the origin and releases a molecule at time $t=0$. Let $X(t)$ denote the position of the released molecule at some specific time $t$. According to [17], under the Brownian motion in three-dimensional space, the probability density of $X(t)$ is

$$
P_{X}(x, t)=\frac{1}{\sqrt{(4 \pi D t)^{3}}} \exp \left(-\frac{x^{2}}{4 D t}\right)
$$

where $x$ represents the distance from the original emission point and $D$ is the aforementioned diffusion constant. Equation (2) illustrates the prorogation model of the molecules in the medium, which can be regarded as the impulse response of the Brownian motion-based channel. Note that Brownian motion has been corroborated to be practical and extensively adopted in the literature, as in $[5,10,17]$. According to the aforementioned amplitude modulation model, only the molecules absorbed by the receiver within time $t_{s}$ are considered as useful ones. Suppose the distance between the transmitter and receiver is $d$ and the radius of the receiver is $R$. Then, according to [15], the probability that a molecule is absorbed by the receiver within $t_{s}$ can be calculated by

$$
P_{a}\left(d, t_{s}\right)=\frac{d}{R} \operatorname{erfc}\left(\frac{d-R}{\sqrt{4 D t_{s}}}\right)
$$

where the error function $\operatorname{erfc}(x)=\frac{2}{\sqrt{\pi}} \int_{x}^{\infty} e^{-u^{2}} d u$. Figure 3 shows the relationship between the absorbing probability $P_{a}$ and the distance $d$ under different lengths of time slot $t_{s}$. We can see that $P_{a}\left(d, t_{s}\right)$ is a decreasing function in terms of distance $d$. This is consistent with the intuition that the farther from the transmitter, the more difficult the receiver can absorb the molecule. On the other hand, the absorbing probability $P_{a}\left(d, t_{s}\right)$ is an increasing function in terms of the length of time slot $t_{s}$. It is also intuitive that the longer the reception time, the higher probability that the molecule can reach the receiver.
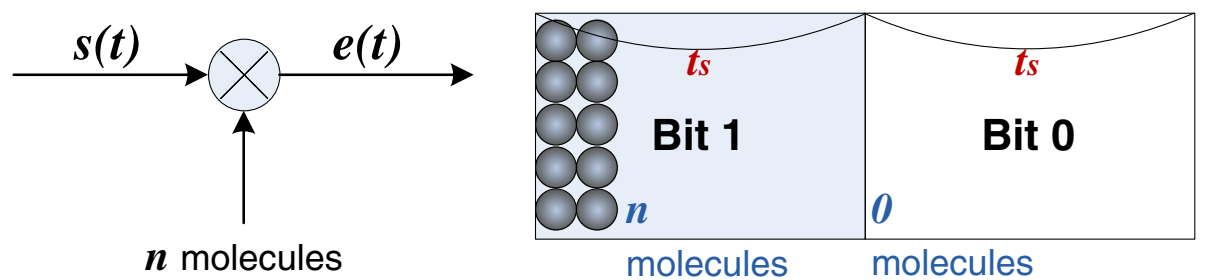

Figure 2 Amplitude modulation. 


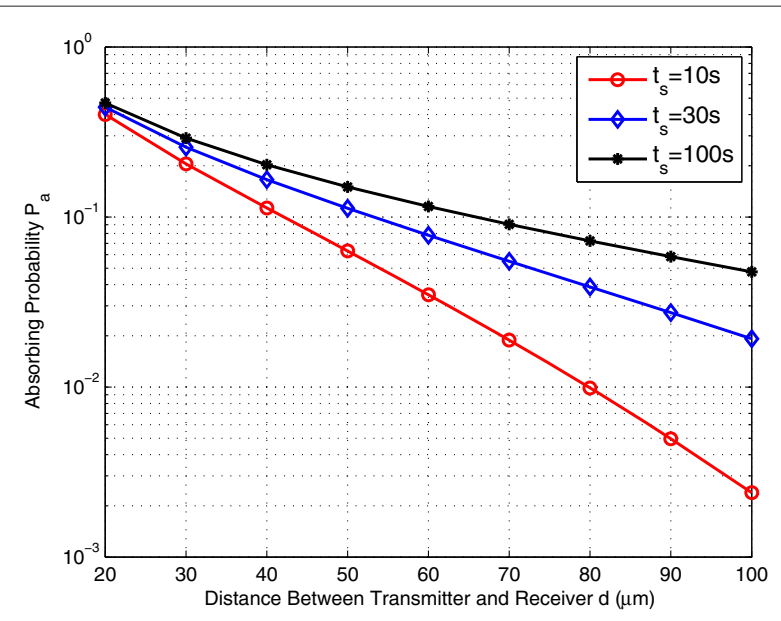

Figure 3 Absorbing probability versus the distance between the transmitter and receiver. The radius of the receiver is $R=10 \mu \mathrm{m}$, and the diffusion coefficient is $D=79.4 \mu \mathrm{m}^{2} / \mathrm{s}$ [29].

\section{Interference analysis}

In this section, we analyze the interference issues in the molecular communication system. Similar to the traditional wireless communication systems, there are inter-symbol interference and inter-user interference in molecular communication systems. For each transmitter, the molecules released in the previous time slots may leak into the current time slot, i.e., the current symbol, which causes the inter-symbol interference (ISI), as shown in the left part of Figure 4. Moreover, the molecules released by one transmitter may also leak into the time slot of other transmitters, which causes the inter-user interference (IUI), as shown in the right part of Figure 4. Based on the interference analysis, we can further derive the optimal decision threshold based on MAP method and the corresponding BER performance.

According to the system model, if the transmitter intends to send bit ' 1 ', it would release $n$ molecules to the medium. Let $N_{a}$ denote the number of molecules absorbed by the receiver located $d$ away from the transmitter within time period $t_{s}$. Since all the molecules are independent of each other and each reaches the receiver with probability $P_{a}\left(d, t_{s}\right), N_{a}$ follows a binomial distribution as follows $[15,33,34]$ :

$$
N_{a} \sim \mathcal{B}\left(n, P_{a}\left(d, t_{s}\right)\right) \text {. }
$$

When $n$ is sufficiently large (e.g., $n \geq 20$ ), which is generally satisfied by the molecular communication systems, the binomial distribution can be approximated by a normal distribution [15,33]. In such a case, the distribution of $N_{a}$ can approximated by

$$
N_{a} \sim \mathcal{N}\left(n P_{a}\left(d, t_{s}\right), n P_{a}\left(d, t_{s}\right)\left(1-P_{a}\left(d, t_{s}\right)\right)\right) .
$$

\subsection{Inter-symbol interference}

The ISI defined in traditional wireless communication systems represents the power leakage of one transmitted symbol into the following symbols, which is usually caused by multi-path propagation. Similarly, in the molecular communication system, due to the random nature of the Brownian motion-based diffusion process, molecules emitted in previous time slots may arrive at the receiver in the current time slot, which leads to the interference to the current bit reception. Such interference is defined as ISI in molecular communications, as shown in the left part of Figure 4. Suppose that the current reception time slot is $m$; let us denote $N_{a, i t_{s}}$ as the number of molecules
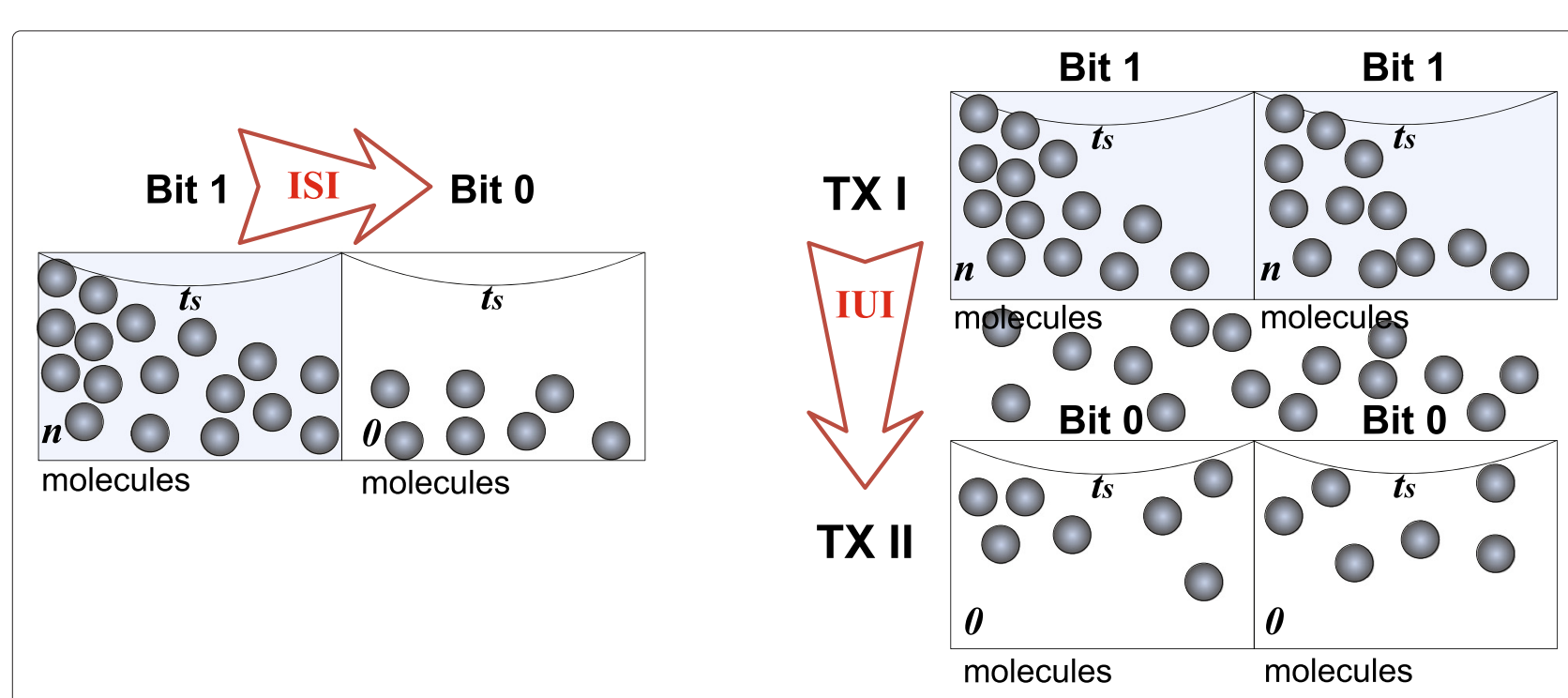

Figure 4 Inter-symbol interference and inter-user interference illustration. 
that were sent at the beginning of $i$ time slots before, i.e., at $(m-i) t_{s}$, and leak into the current time slot $m$. In such a case, $N_{a, i t_{s}}$ should follow the distribution as follows:

$$
\begin{aligned}
N_{a, i t_{s}} \sim & \frac{1}{2} \mathcal{N}\left(n P_{a}\left(d,(i+1) t_{s}\right), n P_{a}\left(d,(i+1) t_{s}\right)\right. \\
& \left.\times\left(1-P_{a}\left(d,(i+1) t_{s}\right)\right)\right) \\
& -\frac{1}{2} \mathcal{N}\left(n P_{a}\left(d, i t_{s}\right), n P_{a}\left(d, i t_{s}\right)\left(1-P_{a}\left(d, i t_{s}\right)\right)\right) .
\end{aligned}
$$

where $\frac{1}{2}$ is based on the assumption that bits ' 1 ' and ' 0 ' are equally generated; the first term represents the total number of molecules that were sent at $m t_{s}-i t_{s}$ and absorbed by the receiver within all subsequent $i+1$ time slots, while the second term represents that within all subsequent $i$ time slots, both of which follow the normal distribution as defined in (5). Thus, we can have the distribution of total interference caused by all previous time slots, $N_{\text {ISI }}$, as follows:

$$
\begin{aligned}
N_{\mathrm{ISI}}= & \sum_{i=1}^{\infty} N_{a, i t_{s}} \sim \sum_{i=1}^{\infty} \frac{1}{2} \mathcal{N}\left(n P_{a}\left(d,(i+1) t_{s}\right)\right. \\
& \left.n P_{a}\left(d,(i+1) t_{s}\right)\left(1-P_{a}\left(d,(i+1) t_{s}\right)\right)\right) \\
& -\sum_{i=1}^{\infty} \frac{1}{2} \mathcal{N}\left(n P_{a}\left(d, i t_{s}\right), n P_{a}\left(d, i t_{s}\right)\left(1-P_{a}\left(d, i t_{s}\right)\right)\right)
\end{aligned}
$$

According to [33], the experiment results have shown that only the interference from one previous time slot, i.e., the ISI from the last symbol, needs to be considered. In such a case, the distribution of ISI $N_{\text {ISI }}$, can be approximated by

$$
\begin{aligned}
N_{\text {ISI }} \sim & \frac{1}{2} \mathcal{N}\left(n P_{a}\left(d, 2 t_{s}\right), n P_{a}\left(d, 2 t_{s}\right)\left(1-P_{a}\left(d, 2 t_{s}\right)\right)\right) \\
& -\frac{1}{2} \mathcal{N}\left(n P_{a}\left(d, t_{s}\right), n P_{a}\left(d, t_{s}\right)\left(1-P_{a}\left(d, t_{s}\right)\right)\right) .
\end{aligned}
$$

\subsection{Inter-user interference}

The IUI defined in traditional wireless communication systems represents the interference power from one transmitter to the other. Similarly, in molecular communications, when there are two or more transmitters releasing molecules into the medium, they would interfere each other at the receiver side, as shown in the right part of Figure 4. Let us consider the two-transmitter case as shown in Figure 1, where the receiver is located $d_{1}$ away from transmitter (TX) I and $d_{2}$ away from TX II. The emission power of TX I is $n_{1}$ and that of TX II is $n_{2}$, i.e., releasing $n_{1}$ or $n_{2}$ molecules to represent bit ' 1 '. Similar to the aforementioned ISI analysis, the IUI for TX I is caused by the molecules which leaked from TX II, and vice versa.
For the symbol of TX II transmitted at time slot $m$, the molecules leaked to it should be considered from both the previous symbol at time slot $m-1$ and the current symbol of TX I. There are four cases: '00', '01', '10', and '11', where ' 10 ', for example, means TX I transmitted ' 1 ' in the previous time slot and transmits ' 0 ' in the current time slot.

For case '00', there is no IUI since no molecule is released. For case ' 01 ', only the current symbol ' 1 ' of TX I causes interference to TX II. In this case, the number of molecules that leaks into the current symbol of TX II from TX I, denoted by $N_{01}^{\mathrm{I}}$, follows normal distribution as

$$
\begin{aligned}
N_{01}^{\mathrm{I}} & \sim \mathcal{N}\left(n_{1} P_{a}\left(d_{1}, t_{s}\right), n_{1} P_{a}\left(d_{1}, t_{s}\right)\left(1-P_{a}\left(d_{1}, t_{s}\right)\right)\right) \\
& =\mathcal{N}\left(n_{1} P_{1 c}, n_{1} P_{1 c}\left(1-P_{1 c}\right)\right),
\end{aligned}
$$

where $P_{1 c}=P_{a}\left(d_{1}, t_{s}\right)$ defined in (3) represents the absorbing probability of molecules of TX I's current symbol at the current time slot, which is interference to TX II. For case '10', only the previous symbol ' 1 ' of TX I causes interference to TX II. In this case, the number of molecules that leaks into the current symbol of TX II from TX I, denoted by $N_{10}^{\mathrm{I}}$, also follows normal distribution as

$$
\begin{aligned}
N_{10}^{\mathrm{I}} \sim & \mathcal{N}\left(n_{1} P_{a}\left(d_{1}, 2 t_{s}\right), n_{1} P_{a}\left(d_{1}, 2 t_{s}\right)\left(1-P_{a}\left(d_{1}, 2 t_{s}\right)\right)\right) \\
& -\mathcal{N}\left(n_{1} P_{a}\left(d_{1}, t_{s}\right), n_{1} P_{a}\left(d_{1}, t_{s}\right)\left(1-P_{a}\left(d_{1}, t_{s}\right)\right)\right) \\
= & \mathcal{N}\left(n_{1} P_{1 p}, n_{1} P_{1 p}\left(1-P_{1 p}\right)\right) \\
& -\mathcal{N}\left(n_{1} P_{1 c}, n_{1} P_{1 c}\left(1-P_{1 c}\right)\right),
\end{aligned}
$$

where $P_{1 p}=P_{a}\left(d_{1}, 2 t_{s}\right)$ represents the absorbing probability of molecules of TX I's previous symbol at both the previous and current time slots. Note that the IUI in case ' 10 ' is quite similar to the ISI in (8), while the difference is that ISI means the interference from the transmitter's own previous symbol, while IUI in case ' 10 ' means the interference from the other transmitter's previous symbol. For case ' 11 ', both the previous and current symbols of TX I can cause interference to TX II. Thus, the number of molecules that leaks into the current symbol of TX II from TX I, denoted by $N_{11}^{\mathrm{I}}$, follows another normal distribution as

$$
\begin{aligned}
N_{11}^{\mathrm{I}} & \sim \mathcal{N}\left(n_{1} P_{1 c}, n_{1} P_{1 c}\left(1-P_{1 c}\right)\right)+\mathcal{N}\left(n_{1} P_{1 p}, n_{1} P_{1 p}\left(1-P_{1 p}\right)\right) \\
& -\mathcal{N}\left(n_{1} P_{1 c}, n_{1} P_{1 c}\left(1-P_{1 c}\right)\right) .
\end{aligned}
$$

Overall, based on the assumption that the probabilities of all the four cases are identical, i.e., $P_{00}=P_{01}=P_{10}=$ 
$P_{11}=\frac{1}{4}$, the IUI from TX I to TX II, denoted by $N_{\mathrm{IUI}}^{\mathrm{I}}$, follows the distribution as follows:

$$
\begin{aligned}
N_{\mathrm{IUI}}^{\mathrm{I}} & =\frac{1}{4}\left(N_{00}^{\mathrm{I}}+N_{01}^{\mathrm{I}}+N_{10}^{\mathrm{I}}+N_{11}^{\mathrm{I}}\right) \\
& \sim \frac{1}{4} \mathcal{N}\left(2 n_{1} P_{1 p}, 4 n_{1} P_{1 c}\left(1-P_{1 c}\right)+2 n_{1} P_{1 p}\left(1-P_{1 p}\right)\right)
\end{aligned}
$$

Similarly, the IUI from TX II to TX I, denoted by $N_{\text {IUI }}^{\mathrm{II}}$, follows the distribution as follows:

$N_{\mathrm{IUI}}^{\mathrm{II}} \sim \frac{1}{4} \mathcal{N}\left(2 n_{2} P_{2 p}, 4 n_{2} P_{2 c}\left(1-P_{2 c}\right)+2 n_{2} P_{2 p}\left(1-P_{2 p}\right)\right)$

where $P_{2 c}=P_{a}\left(d_{2}, t_{s}\right)$ and $P_{2 p}=P_{a}\left(d_{2}, 2 t_{s}\right)$ can be calculated by (3). Note that multiplexing techniques can be adopted to alleviate the IUI, which is not the focus of this paper. The techniques from traditional wireless communications may be used to improve the multi-access performance. For examples, the time-division multiplexing techniques can be easily applied in molecular communications, where the molecular transmissions are scheduled by time slots and by utilizing the Neural Delay Boxes (NDBs) connecting different molecular transmitters to the shared medium [35]. Moreover, the space multiplexing techniques can also be applied in molecular multiple access, e.g., MIMO communication based on molecular diffusion [36]. The IUI analysis in this paper can be easily extended to the multi-transmitter scenario. When there are $K$ transmitters, one transmitter may be interfered by other $K-1$ transmitters. Since each transmitter is with four cases: ' 00 ', '01', ' 10 ', and ' 11 ', there are totally $4(K-1)$ cases. Since the interference is additive, there are totally $4(K-1)$ summations in (11). Therefore, the complexity would be linear with the number of transmitters, i.e., $\mathcal{O}(K)$

\subsection{Bit error rate performance}

BER is defined as the number of bit errors divided by the total number of transmitted bits during an evaluated time interval, which is usually approximated by the bit error probability, i.e., the probability that bit ' 0 ' or ' 1 ' is wrongly decoded by the receiver. In the amplitude modulation-based system, the receiver compares the number of molecules absorbed in one time slot with some predefined threshold $\tau$ to decode the bit. In such a case, the BER performance is heavily related with the threshold $\tau$. Therefore, we need to first derive the optimal $\tau$ that minimizes the BER of each transmitter.

Let us consider the two-transmitter case in Figure 1. When TX I transmits bit ' 0 ', i.e., no molecule is released, the number of molecules absorbed by the receiver within current time slot, denoted by $N_{0}^{\mathrm{I}}$, includes both ISI from its previous symbol and IUI from TX II, which follows normal distribution as follows:

$$
N_{0}^{\mathrm{I}}=N_{\mathrm{ISI}}^{\mathrm{I}}+N_{\mathrm{IUI}}^{\mathrm{II}} \sim \mathcal{N}\left(\mu_{0, \mathrm{I}}, \sigma_{0, \mathrm{I}}^{2}\right),
$$

where $N_{\text {ISI }}^{\mathrm{I}}$ can be calculated by $(8)$ and $\mu_{0, \mathrm{I}}, \sigma_{0, \mathrm{I}}^{2}$ are

$$
\begin{aligned}
\mu_{0, \mathrm{I}}= & \frac{1}{2}\left(n_{1} P_{1 p}-n_{1} P_{1 c}+n_{2} P_{2 p}\right), \\
\sigma_{0, \mathrm{I}}^{2}= & \frac{1}{4} n_{1} P_{1 c}\left(1-P_{1 c}\right)+\frac{1}{4} n_{1} P_{1 p}\left(1-P_{1 p}\right) \\
& +\frac{1}{4} n_{2} P_{2 c}\left(1-P_{2 c}\right)+\frac{1}{8} n_{2} P_{2 p}\left(1-P_{2 p}\right) .
\end{aligned}
$$

On the other hand, when TX I transmits bit ' 1 ', i.e., $n_{1}$ molecules are released, the number of molecules absorbed by the receiver within current time slot, denoted by $N_{1}^{\mathrm{I}}$, follows another normal distribution as follows:

$$
N_{1}^{\mathrm{I}}=\mathcal{N}\left(n_{1} P_{1 c}, n_{1} P_{1 c}\left(1-P_{1 c}\right)\right)+N_{0}^{\mathrm{I}} \sim \mathcal{N}\left(\mu_{1, \mathrm{I}}, \sigma_{1, \mathrm{I}}^{2}\right),
$$

where the first term represents the number of molecules absorbed by the receiver among the $n_{1}$ molecules released by TX I, and $\mu_{1, \mathrm{I}}, \sigma_{1, \mathrm{I}}^{2}$ are

$$
\begin{aligned}
\mu_{1, \mathrm{I}}= & \frac{1}{2}\left(n_{1} P_{1 p}+n_{1} P_{1 c}+n_{2} P_{2 p}\right), \\
\sigma_{1, \mathrm{I}}^{2}= & \frac{1}{4} n_{1} P_{1 c}\left(1-P_{1 c}\right)+\frac{5}{4} n_{1} P_{1 p}\left(1-P_{1 p}\right) \\
& +\frac{1}{4} n_{2} P_{2 c}\left(1-P_{2 c}\right)+\frac{1}{8} n_{2} P_{2 p}\left(1-P_{2 p}\right) .
\end{aligned}
$$

At the receiver side, to demodulate TX I's information, it is confronted with the following hypothesis test based on the absorbed molecules $Y$.

$$
\begin{aligned}
& \mathcal{H}_{0}: \quad Y=N_{0}^{\mathrm{I}} \sim \mathcal{N}\left(\mu_{0, \mathrm{I}}, \sigma_{0, \mathrm{I}}^{2}\right) \\
& \mathcal{H}_{1}: \quad Y=N_{1}^{\mathrm{I}} \sim \mathcal{N}\left(\mu_{1, \mathrm{I}}, \sigma_{1, \mathrm{I}}^{2}\right) .
\end{aligned}
$$

With the hypothesis test model above, we can derive the optimal decision that minimizes the BER using the MAP detection method. Since we assume that bits ' 0 ' and ' 1 ' are uniformly distributed, i.e., $p\left(\mathcal{H}_{0}\right)=p\left(\mathcal{H}_{1}\right)$, the MAP test is equivalent to the the likelihood-ratio test (LRT) as follows:

$$
\begin{aligned}
\frac{p\left(Y \mid \mathcal{H}_{1}\right)}{p\left(Y \mid \mathcal{H}_{0}\right)}= & \frac{\sigma_{0, \mathrm{I}}}{\sigma_{1, \mathrm{I}}} \exp \left\{-\frac{\left(Y-\mu_{1, \mathrm{I}}\right)^{2}}{2 \sigma_{1, \mathrm{I}}^{2}}+\frac{\left(Y-\mu_{0, \mathrm{I}}\right)^{2}}{2 \sigma_{0, \mathrm{I}}^{2}}\right\} \\
= & \frac{\sigma_{0, \mathrm{I}}}{\sigma_{1, \mathrm{I}}} \exp \left\{-\frac{1}{2}\left(\frac{1}{\sigma_{1, \mathrm{I}}^{2}}-\frac{1}{\sigma_{0, \mathrm{I}}^{2}}\right) Y^{2}\right. \\
& \left.+\left(\frac{\mu_{1, \mathrm{I}}}{\sigma_{1, \mathrm{I}}^{2}}-\frac{\mu_{0, \mathrm{I}}}{\sigma_{0, \mathrm{I}}^{2}}\right) Y-\frac{1}{2}\left(\frac{\mu_{1, \mathrm{I}}^{2}}{\sigma_{1, \mathrm{I}}^{2}}-\frac{\mu_{0, \mathrm{I}}^{2}}{\sigma_{0, \mathrm{I}}^{2}}\right)\right\} .
\end{aligned}
$$


By setting the LRT test $\frac{p\left(Y \mid \mathcal{H}_{1}\right)}{p\left(Y \mid \mathcal{H}_{0}\right)}$ as 1 , we can find the MAP decision threshold for demodulating information from TX I as

$$
\tau_{1}=\operatorname{round}\left(\frac{\sqrt{2 A \ln C+B^{2}}-B}{A}\right),
$$

where round represents the rounding operation since the decision threshold should be an integer; the parameters $A$, $B$, and $C$ are as follows:

$$
\begin{aligned}
& A=-\left(\frac{1}{\sigma_{1, \mathrm{I}}^{2}}-\frac{1}{\sigma_{0, \mathrm{I}}^{2}}\right), \quad B=\frac{\mu_{1, \mathrm{I}}}{\sigma_{1, \mathrm{I}}^{2}}-\frac{\mu_{0, \mathrm{I}}}{\sigma_{0, \mathrm{I}}^{2}}, \\
& C=\frac{\sigma_{1, \mathrm{I}}^{2}}{\sigma_{0, \mathrm{I}}^{2}} \exp \left\{\frac{1}{2}\left(\frac{\mu_{1, \mathrm{I}}^{2}}{\sigma_{1, \mathrm{I}}^{2}}-\frac{\mu_{0, \mathrm{I}}^{2}}{\sigma_{0, \mathrm{I}}^{2}}\right)\right\} .
\end{aligned}
$$

Based on the optimal decision threshold, we can calculate the BER of information from TX I as follows:

$$
\begin{aligned}
P_{e}^{\mathrm{I}} & =\frac{1}{2}\left(P\left(N_{0}^{\mathrm{I}} \geq \tau_{1}\right)+P\left(N_{1}^{\mathrm{I}} \leq \tau_{1}\right)\right) \\
& =\frac{1}{2}\left(1-Q\left(\frac{\tau_{1}-\mu_{1, \mathrm{I}}}{\sigma_{1, \mathrm{I}}}\right)+Q\left(\frac{\tau_{1}-\mu_{0, \mathrm{I}}}{\sigma_{0, \mathrm{I}}}\right)\right),
\end{aligned}
$$

where $Q(x)=\frac{1}{\sqrt{2 \pi}} \int_{x}^{\infty} e^{-\frac{u^{2}}{2}} d u$. Similarly, we can derive the BER of information from TX II as

$$
P_{e}^{\mathrm{II}}=\frac{1}{2}\left(1-Q\left(\frac{\tau_{2}-\mu_{1, \mathrm{II}}}{\sigma_{1, \mathrm{II}}}\right)+Q\left(\frac{\tau_{2}-\mu_{0, \mathrm{II}}}{\sigma_{0, \mathrm{II}}}\right)\right)
$$

where $\tau_{2}, \mu_{0, \mathrm{II}}, \mu_{1, \mathrm{II}}, \sigma_{0, \mathrm{II}}$, and $\sigma_{1, \mathrm{II}}$ are dual with $\tau_{1}, \mu_{0, \mathrm{I}}$, $\mu_{1, \mathrm{I}}, \sigma_{0, \mathrm{I}}$, and $\sigma_{1, \mathrm{I}}$; hence, the detailed expressions are omitted here. In Figure 5, we plot the relationships between $P_{e}^{\mathrm{I}}, P_{e}^{\mathrm{II}}$ and $n_{1}$ and $n_{2}$, respectively. From Figure $5 \mathrm{a}$, we can see that TX I's BER $P_{e}^{\mathrm{I}}$ is a decreasing function in terms of $n_{1}$ and an increasing function in terms of $n_{2}$. This is consistent with traditional wireless communication systems that the higher emission power TX I adopts, i.e., the more molecules, $n_{1}$, are transmitted to represent bit ' 1 ', the lower BER performance can be achieved. On the other hand, when TX II enhances its emission power $n_{2}$, the interference to TX I increases, due to which the BER performance of TX I is degraded. For TX II's BER performance shown in Figure 5b, an opposite phenomenon can be found, where $P_{e}^{\mathrm{II}}$ is a decreasing function in terms of its own emission power $n_{2}$, while an increasing function in terms of TX I's emission power $n_{1}$. Thus, an important problem in molecular communication systems is how to control the transmitters' emission power to achieve not only efficiency but also fairness. In the next section, we will solve this technical problem using game theory.

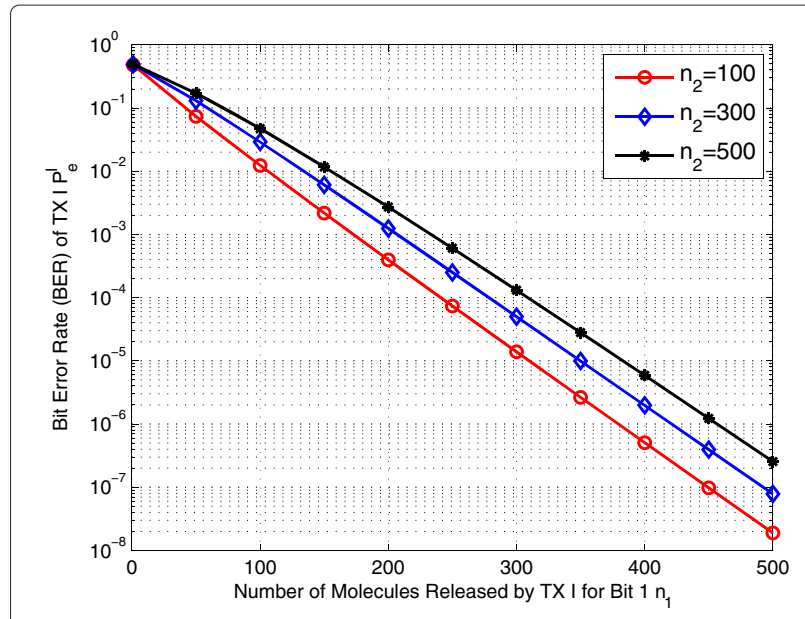

(a)

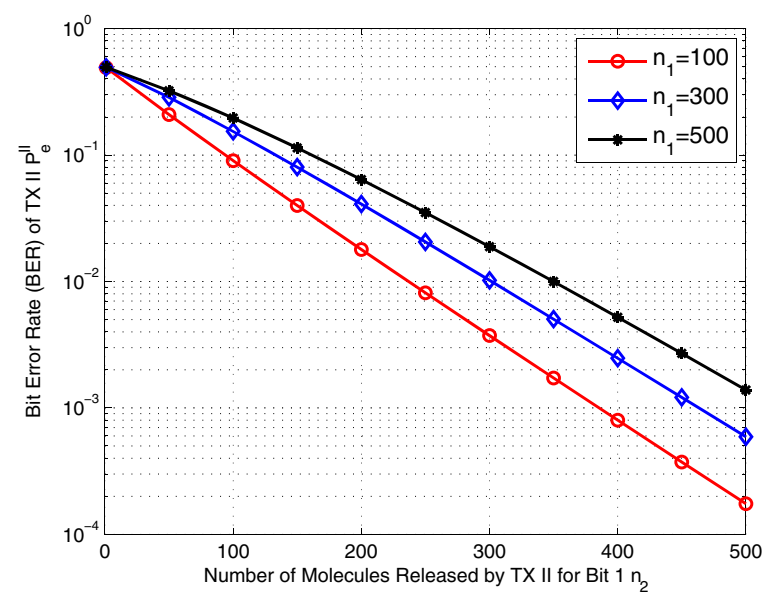

(b)

Figure $\mathbf{5}$ Bit error rate performance. The radius of the receiver is $R=10 \mu \mathrm{m}$. The distances between TX I, TX II and the receiver are $d_{1}=30 \mu \mathrm{m}$ and $d_{2}=40 \mu \mathrm{m}$. The diffusion coefficient is

$D=79.4 \mu \mathrm{m}^{2} / \mathrm{s}$ [29]. (a) Bit error rate of TX I. (b) Bit error rate of TX II.

\section{Molecule emission control game}

In this section, we discuss how to control the molecule emission for both transmitters in Figure 1, i.e., how to control $n_{1}$ for TX I and how to control $n_{2}$ for TX II. As discussed in the introduction, the centralized method can globally optimize all the transmitters' molecule emission by minimizing the total BER $P_{e}^{\mathrm{I}}+P_{e}^{\mathrm{II}}$ derived in (26) and (27). However, the centralized method needs a nanoscale server/coordinator to collect each transmitter's location information, and each coordinator responds with the optimal emission control scheme, which inevitably incurs huge communication and energy cost for the nanoscale molecular machines. Moreover, minimizing the total BER $P_{e}^{\mathrm{I}}+P_{e}^{\mathrm{II}}$ may lead to unfairness phenomenon, e.g., one transmitter is with extremely low BER and the other is with extremely high BER. In the following, we first discuss 
a non-cooperative game formulation of the emission control problem and then consider a cooperative game formulation based on Nash bargaining to improve the system efficiency and ensure the fairness between TX I and TX II. The Nash bargaining solution shows its merits of low computational cost, low information exchange cost, and proportional fairness.

\subsection{Non-cooperative game formulation}

The game theory models strategic interactions among players using formalized incentive structures. It not only provides game models for an efficient self-enforcing distributed design, but also derives well-defined equilibrium criteria to study the optimality of game outcomes for various game scenarios (static or dynamic, complete or incomplete information, non-cooperative or cooperative) [37]. Generally speaking, a game in the strategic form has three elements: $\mathcal{G}=\langle\mathcal{P}, \mathbf{S}, \mathbf{U}\rangle$, where $\mathcal{P}=\{1,2, \ldots\}$ represents a set of players, $\mathbf{S}=\left\{\mathcal{S}_{1}, \mathcal{S}_{2}, \ldots\right\}$ represents a tuple containing the strategy spaces of all players, and $\mathbf{U}=$ $\left\{U_{1}, U_{2}, \ldots\right\}$ represents a tuple containing payoff functions of all players, which measures the outcome of each player. In a fully distributed molecular communication system, each transmitter has the incentive to minimize the BER of its released information at the receiver. In such a case, we can model the non-cooperative molecule emission control game between TX I and TX II, $\mathcal{G}_{c}=$ $\left\langle\mathcal{P}_{c}, \mathbf{S}_{c}, \mathbf{U}_{c}\right\rangle$, as follows:

- Player: The players of $\mathcal{G}_{c}$ are TX I and TX II in the system, i.e.,

$$
\mathcal{P}_{c}=\{\mathrm{TX} \mathrm{I}, \mathrm{TX} \mathrm{II}\} .
$$

- Strategy: The strategy for each transmitter is defined as its molecules emission power: $n_{1}$ for TX I and $n_{2}$ for TX II, i.e., how many molecules are released to represent bit ' 1 '. We can see that the strategy space of each transmitter is finite and discrete. Suppose the molecule emission constraints for TX I and TX II are $N_{\max }^{\mathrm{I}}$ and $N_{\max }^{\mathrm{II}}$. Thus, the strategy spaces of TX I and TX II, denoted by $\mathcal{S}_{1}$ and $\mathcal{S}_{2}$, respectively, are as follows:

$$
\mathbf{S}_{c}=\left\{\mathcal{S}_{1}, \mathcal{S}_{2}\right\}, \quad \text { where }\left\{\begin{array}{l}
\mathcal{S}_{1}=\left\{0,1, \ldots, N_{\max }^{\mathrm{I}}\right\}, \\
\mathcal{S}_{2}=\left\{0,1, \ldots, N_{\max }^{\mathrm{II}}\right\} .
\end{array}\right.
$$

Moreover, the pure strategy profile of game $\mathcal{G}_{c}$, which is defined as a set of strategies including each player's specified action, can be written as $\left(n_{1}, n_{2}\right)$ [38].

- Utility function: The utility function should be defined proportional to the player's benefit. Since each transmitter expects a lower BER, the utility should be defined inversely proportional to the BER performance. Considering that the worst case of BER is $\frac{1}{2}$ (when each transmitter adopts strategy $n_{1}=n_{2}=0$ and the receiver cannot distinguish between bit ' 1 ' and ' 0 '), we can define the utility function as the difference between $\frac{1}{2}$ and BER to ensure a positive utility. Let $U_{1}$ and $U_{2}$ denote the utility functions of TX I and TX II, respectively, which can be written as follows:

$\mathbf{U}_{c}=\left\{U_{1}, U_{2}\right\}$,

where $\left\{\begin{array}{l}U_{1}=\frac{1}{2}-P_{e}^{\mathrm{I}}=\frac{1}{2}\left(Q\left(\frac{\tau_{1}-\mu_{1, \mathrm{I}}}{\sigma_{1, \mathrm{I}}}\right)-Q\left(\frac{\tau_{1}-\mu_{0, \mathrm{I}}}{\sigma_{0, \mathrm{I}}}\right)\right), \\ U_{2}=\frac{1}{2}-P_{e}^{\mathrm{II}}=\frac{1}{2}\left(Q\left(\frac{\tau_{2}-\mu_{1, \mathrm{II}}}{\sigma_{1, \mathrm{II}}}\right)-Q\left(\frac{\tau_{2}-\mu_{0, \mathrm{II}}}{\sigma_{0, \mathrm{II}}}\right)\right) .\end{array}\right.$

According to the utility function above, we can see that $U_{1}$ is an increasing function in terms of $n_{1}$ and a decreasing function in terms of $n_{2}$. On the other hand, $U_{2}$ is a decreasing function in terms of $n_{1}$ but an increasing function in terms of $n_{2}$.

Based on the game model of $\mathcal{G}_{c}=\left\langle\mathcal{P}_{c}, \mathbf{S}_{c}, \mathbf{U}_{c}\right\rangle$ above, we can further find its NE. Since the utility functions of $\mathcal{G}_{c}$ are monotonic in terms of both transmitters' strategies, the NE can be guaranteed to exist and is unique. Apparently, the NE is that each transmitter uses the highest molecule emission power, which would lead to low system efficiency compared with the centralized scheme. Therefore, to improve the system efficiency, we formulate the emission control problem as a cooperative game using the Nash bargaining solution in next subsection.

\subsection{Cooperative game formulation based on Nash bargaining}

In game theory, a cooperative game studies how a group of players should cooperate with each other when noncooperation leads to an unfavorable outcome for each player, e.g., in the non-cooperative molecule emission control game discussed above, each transmitter adopts the highest molecule emission power and heavily interferes with each other. Under such circumstances, the players have the incentive to cooperatively enhance the system efficiency in conjunction with fairness. The bargaining game is one branch of the cooperative game, where two players try to reach an agreement on trading/sharing a limited amount of resources. These two individual players have a choice to bargain with each other so that both of them can gain benefit higher than that without cooperation. In a bargaining game, since there might be an infinite number of social optimal agreement points (i.e., bargaining solutions), three kinds of bargaining solutions egalitarian solution, Kalai-Smorodinsky solution, and Nash bargaining solution [38] - were proposed to refine 
the multiple bargaining solutions, among which the Nash bargaining solution is mostly widely adopted with the emphasis of fairness and social optimality [39]. In the following, we formulate the emission control game between two transmitters as a bargaining game and find the Nash bargaining solution for them.

The emission control bargaining game for molecular communications can be described as follows. Let $\mathcal{P}_{c}=$ $\{$ TX I, TX II $\}$ denote the set of two players, i.e., two nanoscale transmitters. Let $\mathbf{W}=\left\{\left(U_{1}, U_{2}\right)\right\} \subset \mathbb{R}^{2}$ represent the set of feasible payoff allocations that each transmitter can get if they can reach an agreement to cooperate. Let $\mathbf{U}_{\min }=\left(U_{\min }^{\mathrm{I}}, U_{\min }^{\mathrm{II}}\right)$, where $U_{\min }^{\mathrm{I}}$ and $U_{\text {min }}^{\mathrm{II}}$ represent the minimal payoffs that TX I and TX II can be satisfied if both of them cooperate with each other. One way is to set the $U_{\min }^{\mathrm{I}}$ and $U_{\min }^{\mathrm{II}}$ to be the $\mathrm{NE}$ of the non-cooperative molecule emission control game analyzed in the previous subsection. This means that both of the transmitters agree to cooperate only if their respective utility cannot be less than that of the noncooperative scenario. However, in such a case, fairness cannot be guaranteed since the TX, which can obtain a much higher utility in the non-cooperative scenario, can still obtain a much higher utility than the other in this cooperative scenario, which may lead to the failure of cooperation. Therefore, as in most cases, to ensure fairness among transmitters, we set $U_{\min }^{\mathrm{I}}=U_{\text {min }}^{\mathrm{II}}=0$, which means that as long as the utility is larger than 0 , both transmitters would be cooperative. In such a case, all the possible solutions in $\mathbf{W}$ satisfy the individual rationality, i.e., $\left\{\left(U_{1}, U_{2}\right) \mid U_{1} \geq U_{\text {min }}^{\mathrm{I}}, U_{2} \geq U_{\text {min }}^{\mathrm{II}}\right\} \subset \mathbf{W}$ is the set of all the possible bargaining solutions. This is also practical since the NE of the non-cooperative game usually corresponds to the scenario that each transmitter has little utility, i.e., $U_{\min }^{\mathrm{I}} \rightarrow 0, U_{\min }^{\mathrm{II}} \rightarrow 0$. Thus, the pair $\left(\mathbf{W}, \mathbf{U}_{\min }\right)$ can be defined as the emission control bargaining game. We can see that there are numerous possible operating points for TX I and TX II in W. The Nash bargaining solution (NBS), defined as follows, provides a unique, fair and efficient operating point in $\mathbf{W}$ with the idea that after the minimal requirements are satisfied for both transmitters, the rest of the resources are allocated proportionally to each of them according to their conditions.

Definition 1: In the molecule emission control bargaining game $\left(\mathbf{W}, \mathbf{U}_{\text {min }}\right)$, a solution $\mathbf{U}^{*}=\left(U_{1}^{*}, U_{2}^{*}\right)$ is said to be an NBS in $\mathbf{W}$ if the following axioms are satisfied.

1. Pareto optimality: There does not exist a point $\mathbf{U}^{\prime}=\left(U_{1}^{\prime}, U_{2}^{\prime}\right)$ other than $\mathbf{U}^{*}$ in $\mathbf{W}$, satisfying that $U_{1}^{\prime} \geq U_{1}^{*}$ and $U_{2}^{\prime} \geq U_{2}^{*}$.

2. Symmetry: If the feasible set $\mathbf{W}$ is symmetric in terms of two transmitters, for example, when TX I and TX II are homogenous, then $\mathbf{U}^{*}$ is also symmetric, i.e., $U_{1}^{*}=U_{2}^{*}$.
3. Independence of irrelevance alternatives: If $\mathbf{U}^{*} \in \mathbf{W}^{\prime} \subset \mathbf{W}$ is an NBS for $\left(\mathbf{W}, \mathbf{U}_{\text {min }}\right)$, then it is also an NBS for $\left(\mathbf{W}^{\prime}, \mathbf{U}_{\min }\right)$.

4. Independence of linear transformations: For any linear transformation $\psi$, if $\mathbf{U}^{*}$ is an NBS for $\left(\mathbf{W}, \mathbf{U}_{\min }\right)$, then $\psi\left(\mathbf{U}^{*}\right)$ is an NBS for $\left(\psi(\mathbf{W}), \psi\left(\mathbf{U}_{\min }\right)\right)$.

Among the axioms, the physical meaning of Pareto optimality is that there exists no other operating point that can lead to superior performance for one transmitter without degrading the performance of the other. In other words, Pareto optimality ensures that all the system resources have been fully utilized. Axioms 2 to 4 are related to fairness, where the symmetry axiom requires that if the feasible set is completely symmetric, then TX I and TX II have exactly the same utility, the irrelevance alternatives axiom can help to refine the feasible solution set by eliminating the irrelevant ones, and the linear property asserts that the NBS is scale-invariant. We can see that an NBS can not only achieve a high system efficiency, but also guarantee the fairness issue. It has been shown that there is a unique NBS that satisfies all the axioms in Definition 1 [38], wherein the solution maximizes the product of TX I's and TX II's utilities, i.e.,

$\mathbf{U}^{*}=\left(U_{1}^{*}, U_{2}^{*}\right)=\arg \max _{n_{1} \leq N_{\max }^{\mathrm{I}}, n_{2} \leq N_{\max }^{\mathrm{II}}} U_{1}\left(n_{1}, n_{2}\right) \cdot U_{2}\left(n_{1}, n_{2}\right)$.

In such a case, the Nash bargaining equilibrium for both transmitters can be calculated by solving the optimization problem as follows:

$$
\begin{aligned}
\left(n_{1}^{*}, n_{2}^{*}\right)= & \arg \max _{n_{1}, n_{2}} \frac{1}{4}\left(Q\left(\frac{\tau_{1}-\mu_{1, \mathrm{I}}}{\sigma_{1, \mathrm{I}}}\right)-Q\left(\frac{\tau_{1}-\mu_{0, \mathrm{I}}}{\sigma_{0, \mathrm{I}}}\right)\right) \\
& \times\left(Q\left(\frac{\tau_{2}-\mu_{1, \mathrm{II}}}{\sigma_{1, \mathrm{II}}}\right)-Q\left(\frac{\tau_{2}-\mu_{0, \mathrm{II}}}{\sigma_{0, \mathrm{II}}}\right)\right) \\
\text { s.t. } & n_{1} \leq N_{\max }^{\mathrm{I}}, \quad n_{2} \leq N_{\max }^{\mathrm{II}}
\end{aligned}
$$

Since it is difficult to derive closed-form expressions for $U_{1}\left(n_{1}, n_{2}\right)$ and $U_{2}\left(n_{1}, n_{2}\right)$, the optimization problem above has to be numerically solved. Suppose $U\left(n_{1}, n_{2}\right)=$ $U_{1}\left(n_{1}, n_{2}\right) \cdot U_{2}\left(n_{1}, n_{2}\right)$, the NBS can be found by using the gradient descent method as follows:

$n_{1}(t+T)=n_{1}(t)+\lambda\left[U\left(n_{1}(t)+1, n_{2}(t)\right)-U\left(n_{1}(t), n_{2}(t)\right)\right]$,

$n_{2}(t+T)=n_{2}(t)+\lambda\left[U\left(n_{1}(t), n_{2}(t)+1\right)-U\left(n_{1}(t), n_{2}(t)\right)\right]$, 
where $T$ is the updating period, $\lambda$ is an integer step size which can be set as 1 , and $\left[U\left(n_{1}(t)+1, n_{2}(t)\right)-U\left(n_{1}(t)\right.\right.$, $\left.\left.n_{2}(t)\right)\right]$ and $\left[U\left(n_{1}(t), n_{2}(t)+1\right)-U\left(n_{1}(t), n_{2}(t)\right)\right]$ are the approximated partial derivatives of $U\left(n_{1}, n_{2}\right)$ over $n_{1}$ and $n_{2}$, respectively. Note that in the implementation, $U\left(n_{1}, n_{2}\right)$ is not calculated using (30), which, instead, is evaluated by the transmitters. Therefore, they need to exchange their utility $U_{1}(t)$ and $U_{2}(t)$ with each other to find the NBS. In Algorithm 1, we summarize the distributed NBS algorithm. Note that the structure of the algorithm can also be applied into multiple TX and RX scenarios, where an extra time slot is required for multiple transmitters exchanging their utility information, i.e., the transmitters sequentially broadcast their utility in step 3 of Algorithm 1.

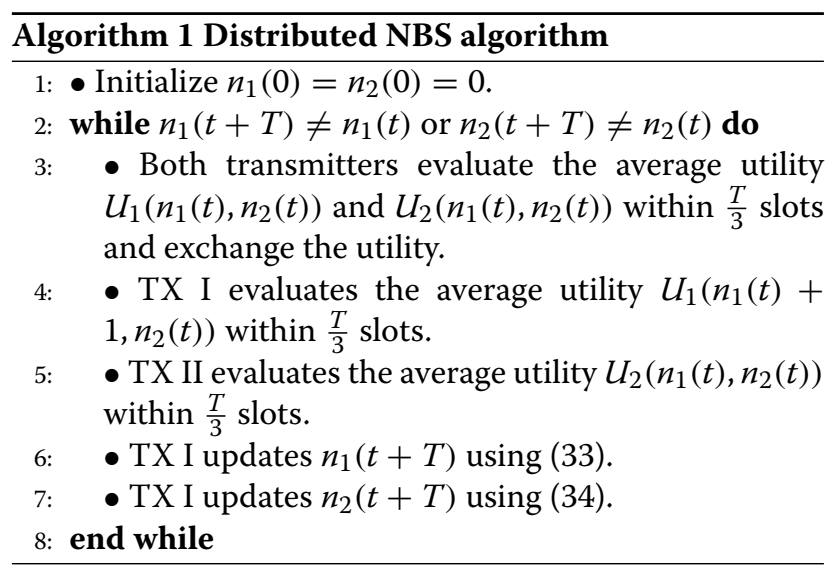

From the definition of NBS, the Pareto optimality quantitatively shows the high efficiency of NBS, while fairness is only illustrated in a qualitative manner. To further quantitatively verify the fairness property of NBS, we introduce the concept of 'proportional fairness', which is a widely used metric to evaluate fairness performance in wired networks [40]. Proportional fairness is a compromise between fairness and performance and based upon maintaining a balance between two competing interests, i.e., trying to maximize the social welfare while at the same time satisfying each user's minimal level of requirement. According to the general definition of proportional fairness [40], the specific definition of proportional fairness in the emission control bargaining game for molecular communications can be described as follows.

Definition 2: In the molecule emission control bargaining game $\left(\mathbf{W}, \mathbf{U}_{\min }\right)$, a solution $\mathbf{U}^{\mathrm{F}}=\left(U_{1}^{\mathrm{F}}, U_{2}^{\mathrm{F}}\right)$ is said to be proportionally fair when any change in $\mathrm{U}^{\mathrm{F}}=\left(U_{1}^{\mathrm{F}}, U_{2}^{\mathrm{F}}\right)$ would result in the sum of proportional changes of the utilities being non-positive, i.e.,

$$
\frac{U_{1}^{\prime}-U_{1}^{\mathrm{F}}}{U_{1}^{\mathrm{F}}}+\frac{U_{2}^{\prime}-U_{2}^{\mathrm{F}}}{U_{2}^{\mathrm{F}}} \leq 0, \quad \forall\left(U_{1}^{\prime}, U_{2}^{\prime}\right) \in \mathbf{W} .
$$

From the definition, we can see that the physical meaning of proportional fairness is that at this point, if one transmitter intends to improve its performance with some increasing percentage, then the other transmitter would suffer from some degrading performance with some decreasing percentage, and the increasing percentage is no larger than the decreasing percentage. Therefore, the Pareto optimality is a special case of proportional fairness. In the following theorem, we will show that the NBS of the emission control bargaining game derived by (31) is a proportionally fair solution.

Since the ln function is concave and monotonic, the optimization problem in (31) is equivalent to the following problem:

$$
\begin{aligned}
\mathbf{U}^{*} & =\left(U_{1}^{*}, U_{2}^{*}\right) \\
& =\arg \max _{n_{1} \leq N_{\max }^{\mathrm{I}}, n_{2} \leq N_{\max }^{\mathrm{II}}} \ln \left(U_{1}\left(n_{1}, n_{2}\right) \cdot U_{2}\left(n_{1}, n_{2}\right)\right) .
\end{aligned}
$$

Let us define $\hat{U}_{1}\left(n_{1}, n_{2}\right)=\ln \left(U_{1}\left(n_{1}, n_{2}\right)\right)$ and $\hat{U}_{2}\left(n_{1}, n_{2}\right)=$ $\ln \left(U_{2}\left(n_{1}, n_{2}\right)\right)$. Thus, the gradients of $\hat{U}_{1}\left(n_{1}, n_{2}\right)$ and $\hat{U}_{2}\left(n_{1}, n_{2}\right)$ at the NBS point $\mathbf{U}^{*}=\left(U_{1}^{*}, U_{2}^{*}\right)$ are $\partial \hat{U}_{1}\left(n_{1}, n_{2}\right) /\left.\partial U_{1}\right|_{U_{1}^{*}}$ and $\partial \hat{U}_{2}\left(n_{1}, n_{2}\right) /\left.\partial U_{2}\right|_{U_{2}^{*}}$. Since the NBS is the unique point that can optimize both (31) and (36), for any point deviating from the NBS point, denoted by $\mathbf{U}^{\prime}=\left(U_{1}^{\prime}, U_{2}^{\prime}\right)$, the following inequality should hold:

$$
\begin{gathered}
\left.\frac{\partial \hat{U}_{1}\left(n_{1}, n_{2}\right)}{\partial U_{1}}\right|_{1}\left(U_{1}^{\prime}-U_{1}^{*}\right)+\left.\frac{\partial \hat{U}_{2}\left(n_{1}, n_{2}\right)}{\partial U_{2}}\right|_{U_{2}^{*}}\left(U_{2}^{\prime}-U_{2}^{*}\right) \leq 0 \\
\Rightarrow \frac{U_{1}^{\prime}-U_{1}^{*}}{U_{1}^{*}}+\frac{U_{2}^{\prime}-U_{2}^{*}}{U_{2}^{*}} \leq 0
\end{gathered}
$$

The first inequality means for all feasible point $\mathbf{U}^{\prime}=$ $\left(U_{1}^{\prime}, U_{2}^{\prime}\right) \in \mathbf{W}$ that is different from NBS point $\mathbf{U}^{*}$, the overall change of two transmitters' utilities is negative according to the gradient.We can see that the second inequality is same as the definition of proportional fairness in (35). Therefore, we can conclude that the NBS of the emission control bargaining game is equivalent to the proportional fairness.

\subsection{Simulation results}

In this subsection, we conduct a simulation to evaluate the performance of the non-cooperative and cooperative game theoretic analyses. In the simulation, the length of each time slot is set as $t_{s}=10 \mathrm{~s}$, the radius of the receiver is configured as $R=10 \mu \mathrm{m}$, and the diffusion coefficient is an empirical parameter set as $D=79.4 \mu \mathrm{m}^{2} / \mathrm{s}$ 


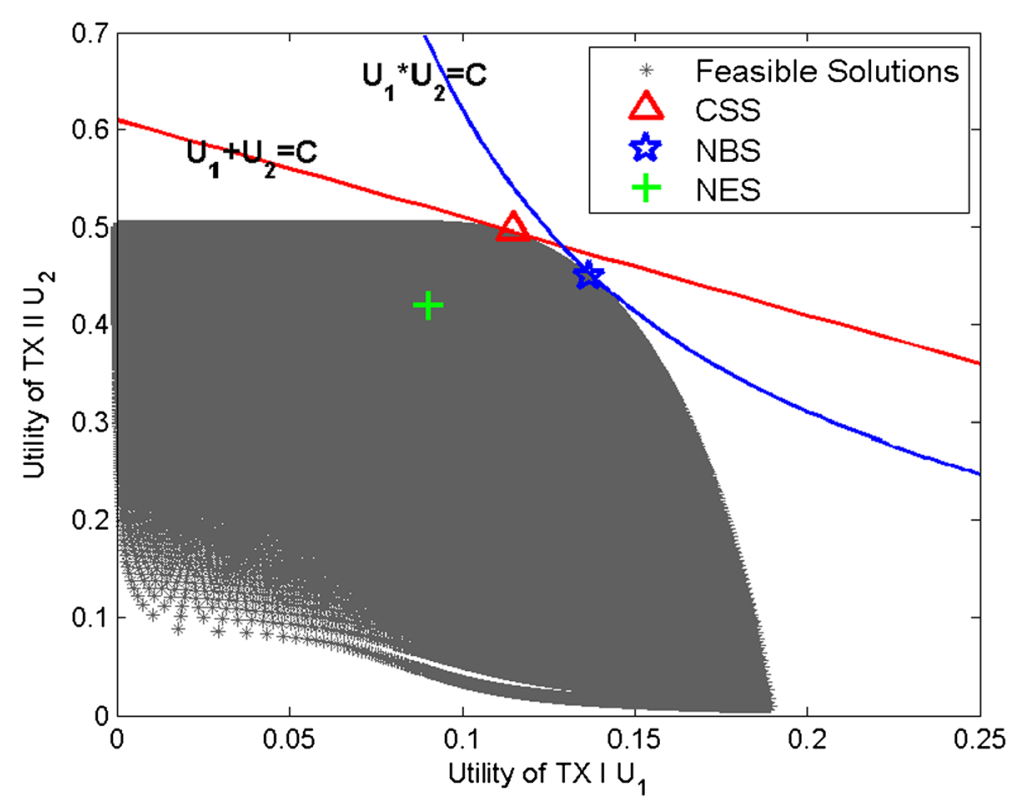

Figure 6 Physical meanings of three solutions.

[29]. As for the location settings, the distance between TX I and the receiver is fixed at $d_{1}=100 \mu \mathrm{m}$, while the distance between TX II and the receiver $d_{2}$ varies from 50 to $150 \mu \mathrm{m}$. The molecule emission constraint for each transmitter is 1,000 , i.e., $N_{\max }^{\mathrm{I}}=N_{\max }^{\mathrm{II}}=1,000$. In the simulation, we compare the performances of three kinds of schemes as follows:

- Centralized scheme solution (CSS): The centralized scheme is to maximize the social welfare of the two transmitters, i.e.,

$$
\max _{n_{1} \leq N_{\max }^{\mathrm{I}}, n_{2} \leq N_{\max }^{\mathrm{II}}} U_{1}\left(n_{1}, n_{2}\right)+U_{2}\left(n_{1}, n_{2}\right) .
$$

- Nash equilibrium solution (NES): Nash equilibrium means the solution of the non-cooperative emission control game, i.e., $n_{1}=N_{\max }^{\mathrm{I}}, n_{2}=N_{\max }^{\mathrm{II}}$.

- Nash bargaining solution (NBS): Nash bargaining means the solution of the cooperative emission control game, i.e.,

$$
\max _{n_{1} \leq N_{\max }^{\mathrm{I}}, n_{2} \leq N_{\max }^{\mathrm{II}}} U_{1}\left(n_{1}, n_{2}\right) \cdot U_{2}\left(n_{1}, n_{2}\right) .
$$

We first conduct a simulation to illustrate the physical meaning of the three schemes as shown in Figure 6 . The dotted area represents all the feasible solutions for $U_{1}$ and

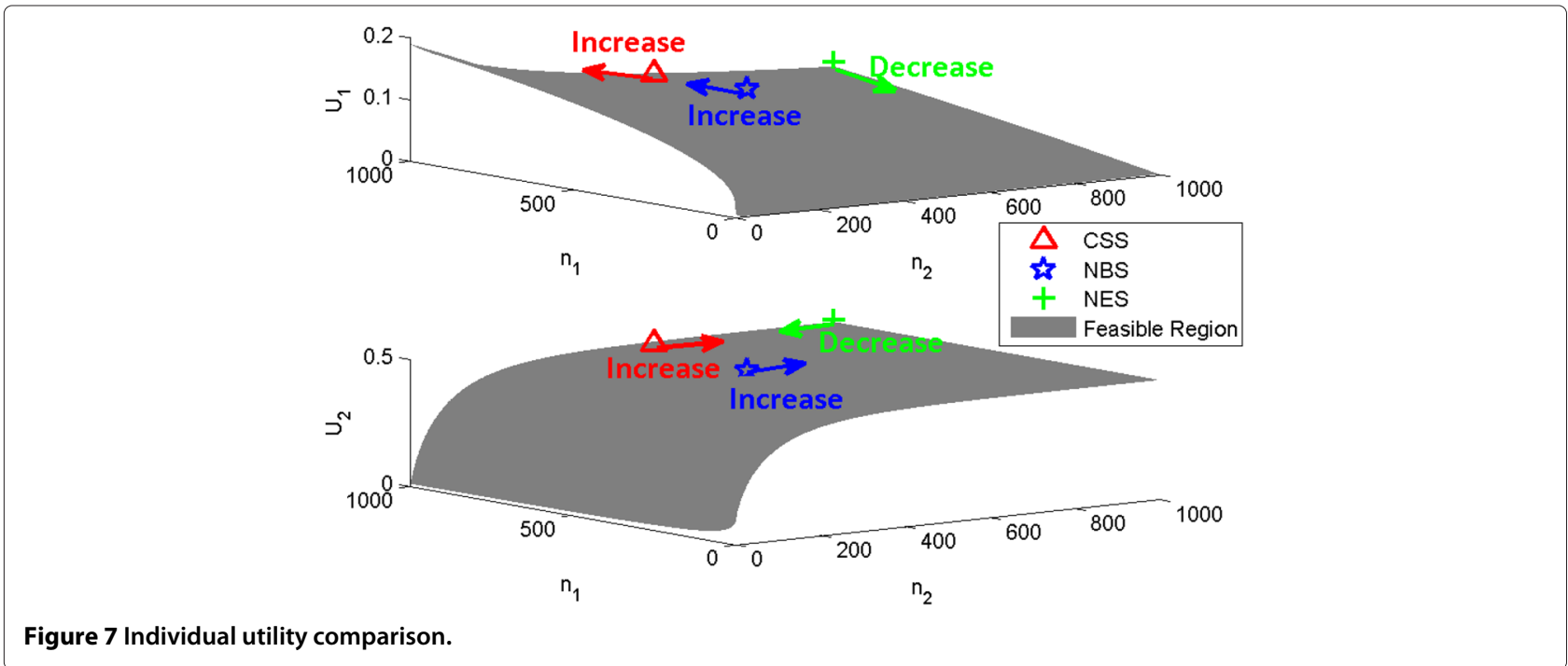




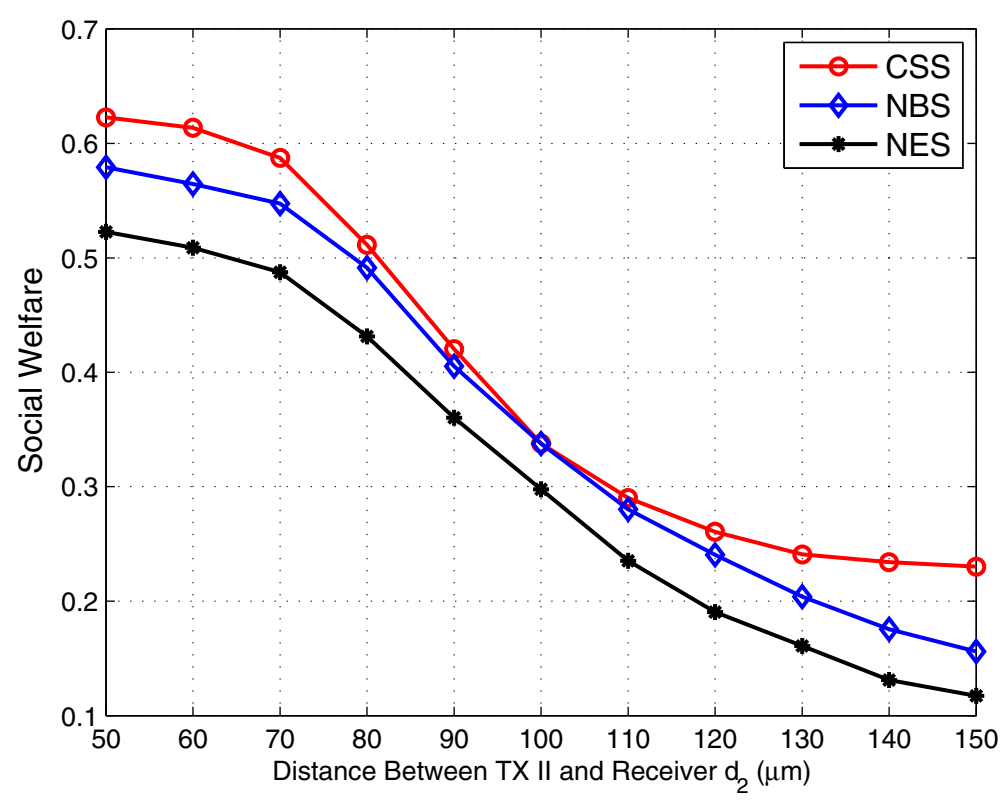

Figure 8 Social welfare comparison.

$U_{2}$ when the location of TX I is $d_{1}=100 \mu \mathrm{m}$ and the location of TX II is $d_{2}=50 \mu \mathrm{m}$. The feasible set is obtained by simulating all possible strategy profiles $\left(n_{1}, n_{2}\right)$, where $1 \leq n_{1} \leq 1,000$ and $1 \leq n_{2} \leq 1,000$ (the cases of $n_{1}=0$ and $n_{1}=0$ are omitted). We can see that for the centralized scheme which maximizes the total utilities, the corresponding solution, i.e., CSS denoted by the red triangle, is just the tangency point between the line
$U_{1}+U_{2}=C$ and the feasible set. For the Nash bargaining scheme that maximizes the product of two transmitters' utilities, the corresponding solution, i.e., NBS denoted by the blue pentagon, is just the tangency point between the line $U_{1} \cdot U_{2}=C$ and the feasible set. Since both transmitters use the highest molecule emission power at the Nash equilibrium solution of the non-cooperative game, their utilities are impaired by the interference, which can

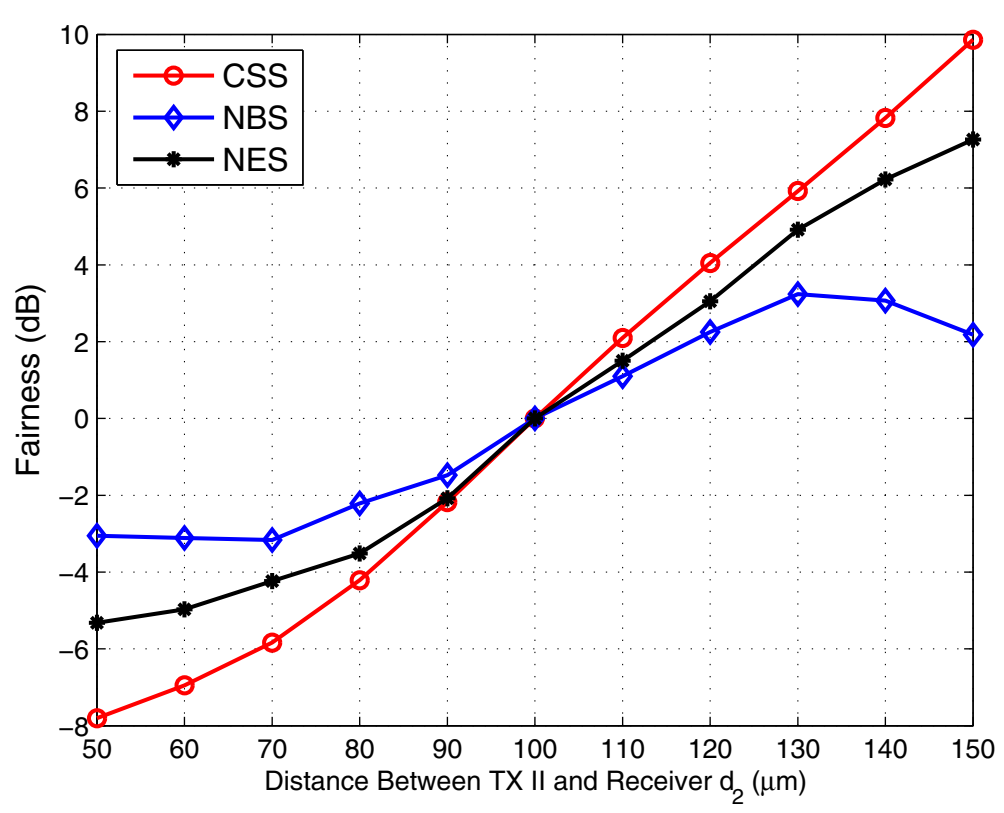

Figure 9 Fairness comparison. 
be seen by the NES denoted by the green plus. However, with NES, no transmitter has the incentive to deviate. As shown in Figure 7, which illustrates the utilities of both transmitters versus their strategies, $n_{1}$ and $n_{2}$, we can see that at the NES denoted by the green plus, there is no deviation direction that can increase either transmitter's utility. While for the CSS and NBS, each transmitter can increase its utility by unilaterally deviating from the current strategy. Therefore, although the NES provides a low system efficiency, it is an equilibrium that neither transmitter will deviate since any deviation will lead to a utility loss.

In Figure 8, we show the total utilities of both transmitters for three schemes versus TX II's location $d_{2}$, i.e., social welfare comparison. We can see that the centralized scheme always has the best performance since its objective function is to maximize the social welfare, while the Nash equilibrium scheme has the worst performance since both transmitters adopt the highest molecule emission power and thus severely interfere with each other. The performance of the Nash bargaining scheme is in the middle, and the gap from the centralized scheme is larger when the two transmitters have very different locations with regard to the receiver, i.e., when $50 \mu \mathrm{m} \leq d_{2} \leq$ $70 \mu \mathrm{m}$ and $130 \mu \mathrm{m} \leq d_{2} \leq 150 \mu \mathrm{m}$ (note that $d_{1}=$ $100 \mu \mathrm{m})$. That is because, in order to balance the utilities between TX I and TX II, the NBS has to sacrifice some system performance to ensure fairness. Moreover, the difference between the two transmitters' utilities is proportional to the difference between their locations relative to the receiver $\left(d_{1}-d_{2}\right)$, which leads to the fact that the larger difference $\left(d_{1}-d_{2}\right)$, the more system performance is required to be compromised in order to guarantee fairness. We can also see that when TX I and TX II are in similar locations, i.e., $90 \mu \mathrm{m} \leq d_{2} \leq 110 \mu \mathrm{m}$, the performances of CSS and NBS are also similar with each other due to the nearly symmetric settings. In addition, to evaluate the fairness performances of those three schemes, we plot the utility ratio of two transmitters in Figure 9, i.e., $10 \log \left(U_{1}\left(n_{1}, n_{2}\right) / U_{2}\left(n_{1}, n_{2}\right)\right)$. Note that the vertical axis is in $\mathrm{dB}$, which means that the closer to $0 \mathrm{~dB}$, the better the fairness performance. Different from the social welfare performance, we can see that the NBS always has the best fairness performance, which further verifies that the NBS is a trade-off between performance and fairness.

\section{Conclusions}

In this paper, we provided a game-theoretic framework for nanoscale molecular communication systems. We analyzed the inter-symbol and inter-user interference, optimal decision threshold, and BER performance in the molecular communication system with amplitude modulation. Based on the interference analysis, we formulated the molecule emission control problem as a non-cooperative emission control game, where the Nash equilibrium was found and proved to be unique. In order to improve the system efficiency while guaranteeing fairness, we further modeled the proposed molecule emission control problem using a cooperative game based on Nash bargaining solution, which was proved to be proportionally fair. Finally, a simulation was conducted to compare the performance among the centralized scheme, Nash equilibrium scheme, and Nash bargaining scheme. The results verified that the NBS can achieve a trade-off between social welfare performance and user fairness.

\section{Competing interests}

The authors declare that they have no competing interests.

\section{Acknowledgements}

This work was funded by projects $61371079,61273214,61271267$, and 91338203 supported by NSFC China, and the Postdoctoral Science

Foundation funded project. The work was done during Chunxiao Jiang's visit to the University of Maryland.

\section{Author details}

${ }^{1}$ Department of Electronic Engineering, Tsinghua University, Beijing 100084, China. ${ }^{2}$ Department of Electrical and Computer Engineering, University of Maryland, College Park, MD 20742, USA.

Received: 25 June 2014 Accepted: 22 December 2014

Published online: 25 January 2015

References

1. IF Akyildiz, J Jornet, The internet of nano-things. IEEE Wireless Commun. 17(6), 58-63 (2010)

2. IF Akyildiz, F Brunetti, C Blazquez, Nanonetworks: a new communication paradigm. Comput. Networks. 52(12), 2260-2279 (2008)

3. S Hiyama, Y Moritani, Molecular communication: harnessing biochemical materials to engineer biomimetic communication systems. Networks. $\mathbf{1}(1), 20-30$ (2010)

4. TNakano, MJ Moore, F Wei, AV Vasilakos, J Shuai, Molecular communication and networking: opportunities and challenges. IEEE Trans. Nanobiosci. 11(2), 135-148 (2012)

5. M Pierobon, IF Akyildiz, A physical end-to-end model for molecular communication in nanonetworks. IEEE J. Sel. Areas Commun. 28(4), 602-611 (2010)

6. SF Walsh, D Balasubramaniam, T Botvich, T Suda, SF Nakano, MO Bush, in Third International Conference on NanoNetworks and Workshops (NanoNet). Foghlu, Hybrid DNA and enzymatic based computation for address encoding, link switching and error correction in molecular communication (USA Boston, MA, September 2008), pp. 28-38

7. TT Nakano, M Suda, R Moore, A Egashira, K Enomoto, in Genetic and Evolutionary Computation Conference (GECCO). Arima, Molecular communication for nanomachines using intercellular calcium signaling (USA Washington D.C., June 2005), pp. 478-481

8. A Eckford, in Information Sciences and Systems (CISS). Nano communication with Brownian motion (USA Baltimore, MD, March 2007), pp. 14-16

9. B Atakan, OB Akan, On channel capacity and error compensation in molecular communication. Trans. Comput. Syst. Biol. X. 5410, 59-80 (2008)

10. B Atakan, OB Akan, Deterministic capacity of information flow in molecular nanonetworks.Nano Commun. Networks. 1(1), 31-42 (2010)

11. D Arifler, Capacity analysis of a diffusion-based short-range molecular nano-communication channel. Comput. Networks. 55(6), 1426-1434 (2011)

12. M A Einolghozati, A Sardari, F Beirami, in IEEE ISIT. Fekri, Capacity of discrete molecular diffusion channels (Saint Petersburg Russia, August 2011), pp. 723-727 
13. S Kadloor, RS Adve, AW Eckford, Molecular communication using Brownian motion with drift. IEEE Trans. NanoBiosci. 11(2), 89-99 (2012)

14. KV Srinivas, AW Eckford, RS Adve, Molecular communication in fluid media: the additive inverse Gaussian noise channel. IEEE Tran. Inform. Theory. 58(7), 4678-4692 (2012)

15. MS Leeson, MD Higgins, Forward error correction for molecular communications.Nano Commun. Networks. 3(1), 161-167 (2012)

16. M Pierobon, IF Akyildiz, Diffusion-based noise analysis for molecular communication in nanonetworks. IEEE Trans. Signal Process. 59(6), 2530-2547 (2011)

17. M Pierobon, IF Akyildiz, in Proc. IEEE ICC. Intersymbol and co-channe interference in diffusion-based molecular communication (Ottawa Canada, June 2012), pp. 6126-6131

18. BD Unluturk, D Malak, OB Akan, Rate-delay tradeoff with network coding in molecular nanonetworks. IEEE Trans. Nanotechnol. 12(2), 120-128 (2013)

19. A Noel, KC Cheung, R Schober, Optimal receiver design for diffusive molecular communication with flow and additive noise. IEEE Trans. NanoBiosci. 13(3), 350-362 (2014)

20. M Pierobon, IF Akyildiz, Noise analysis in ligand-binding reception for molecular communication in nanonetworks. IEEE Trans. Signal Process. 59(9), 4168-4182 (2011)

21. I Karatzas, SE Shreve, Brownian Motion and Stochastic Calculus. (Springer, New York, 1991)

22. MJ Moore, T Suda, K Oiwa, Molecular communication: modeling noise effects on information rate. IEEE Trans. Nanobiosci. 8(2), 169-180 (2009)

23. I I Llatser, N Pascual, A Garralda, M Cabellos-Aparicio, E Pierobon, in Proc IEEE GLOBECOM. Alarcon, Exploring the physical channel of diffusion-based molecular communication by simulation (USA Houston, TX, December 2011), pp. 1-5

24. M A Einolghozati, A Sardari, F Beirami, in Inf. Sci. Syst. (CISS). Fekri, Consensus problem under diffusion-based molecular communication (USA Baltimore, MD, March 2011), pp. 1-6

25. A Einolghozati, FF Mohsen Sardari, in IEEE ISIT. Relaying in diffusion-based molecular communication (Istanbul Turkey, July 2013), pp. 1844-1848

26. Z Han, KJR Liu, Non-cooperative power control game and throughput game over wireless networks. IEEE Trans. Commun. 53(10), 1625-1629 (2005)

27. Z Han, ZJ Ji, KJR Liu, Non-cooperative resource competition game by virtual referee in multi-cell OFDMA. IEEE J. Sel. Areas Commun. 25(6), 1079-1090 (2007)

28. KJR Liu, B Wang, Cognitive Radio Networking and Security: A Game Theoretical View. (Cambridge University Press, Cambridge, 2010)

29. JJ Kim, K Park, Modulated insulin delivery from glucose-sensitive hydrogel dosage forms. J. Controlled Release. 77(1-2), 39-47 (2001)

30. T Zhou, L Chen, K Aihara, Molecular communication through stochastic synchronization induced by extracellular fluctuations. Phys. Rev. Lett. 95(17), 178103 (2005)

31. S Abadal, IF Akyildiz, Automata modeling of quorum sensing for nanocommunication networks. Nano Commun. Networks. 2(1), 74-83 (2011)

32. H ShahMohammadian, GG Messier, S Magierowski, Blind synchronization in diffusion-based molecular communication channels. IEEE Commun. Lett. 17(11), 2156-2159 (2013)

33. MS Kuran, HB Yilmaz, T Tugcu, B Ozerman, Energy model for communication via diffusion in nanonetworks. Nano Commun. Networks. $\mathbf{1}(2), 86-95(2010)$

34. MS Kuran, HB Yilmaz, T Tugcu, IF Akyildiz, Interference effects on modulation techniques in diffusion based nanonetworks. Nano Commun. Networks. 3(1), 65-73 (2012)

35. S H Tezcan, FN Oktug, in IEEE ICC Workshop on Molecular and Nanoscale Communications. Kok, Neural delay lines for TDMA based molecular communication in neural networks (Ottawa Canada, June 2012), pp. 6209-6213

36. P-C L-S Meng, K-C Yeh, IF Chen, Akyildiz, MIMO communications based on molecular diffusion. (CA, Anaheim, December 2012), pp. 5380-5385

37. Z Ji, KJR Liu, Cognitive radios for dynamic spectrum access-dynamic spectrum sharing: a game theoretical overview. IEEE Commun. Mag. 45(5), 88-94 (2007)

38. G Owen, Game Theory. (Academic Press, New York, 2001)
39. Z Han, Z Ji, KJR Liu, Fair multiuser channel allocation for OFDMA networks using Nash bargaining solutions and coalitions. IEEE Trans. Commun. 53(8), 1366-1376 (2005)

40. F Kelly, Charging and rate control for elastic traffic. Eur. Trans. Telecommun. 28(1), 33-37 (1997)

\section{Submit your manuscript to a SpringerOpen ${ }^{\circ}$ journal and benefit from:}

- Convenient online submission

Rigorous peer review

- Immediate publication on acceptance

- Open access: articles freely available online

- High visibility within the field

- Retaining the copyright to your article

Submit your next manuscript at $>$ springeropen.com 